\title{
DETERMINANTES DA OBESIDADE EM CRIANÇAS ACOMPANHADAS POR UMA ENTIDADE DE ASSISTÊNCIA SOCIAL EM ITABUNA-BA
}

\section{DETERMINANTS OF OBESITY IN CHILDREN ACCOMPANIED BY AN ENTITY OF SOCIAL ASSISTANCE IN ITABUNA- BA}

Carla Santos Silva ${ }^{1}$; Maria Ionete Santos Góis²; Marcio Amorim Tolentino Lima3;

\section{Márcia Maria Falcão Sousa 4}

1 Enfermeira - União Metropolitana de Educação e Cultura, UNIME -Itabuna desde 2017. karlynha.live@hotmail.com

2 Enfermeira - União Metropolitana de Educação e Cultura, UNIME -Itabuna desde 2017.netygoes@hotmail.com

${ }^{3}$ Biólogo. Mestre em sistemas aquáticos tropicais, pós-graduando em fisiologia humana aplica às ciências da saúde, docente do ciclo básico e farmácia da União Metropolitana de Educação e Cultura, UNIME desde 2011. marcioatl@yahoo.com.br 4 Nutricionista. Universidade Estadual de Santa Cruz. Docente na união metropolitana de educação e cultura UNIME.

\section{Endereço para correspondência}

Av. J. S. Pinheiro, 1600, Lomanto - Campus I Av. J. S. Pinheiro, 1191, Lomanto Campus II. (73)2102-3030 


\title{
RESUMO
}

A obesidade infantil apresentou um considerável aumento nas últimas décadas, constituindo-se como uma verdadeira pandemia. Trata-se de um quadro preocupante, pois é uma pré-disposição para o desenvolvimento de doenças como a hipertensão, diabetes mellitus tipo 2, doenças cardiovasculares, entre outras. Dado isso buscou-se destacar a importância da qualidade de vida na prevenção da obesidade em menores assistidos por uma entidade de assistência social na cidade de Itabuna-BA, e especificamente identificar o índice de crianças que praticam alguma modalidade de esporte, conhecer a qualidade da alimentação oferecida em domicílio e observar o nível de conhecimento acerca do assunto. Foram avaliadas fichas, em que 25 pais ou responsáveis representaram seus filhos. Ao término da pesquisa, identificou-se que 0 índice de crianças que praticam alguma modalidade de esporte foi de $84 \%$, com um total de $76 \%$ recebendo incentivo à prática desportiva. Quanto à qualidade da alimentação oferecida por seus pais ou responsáveis, no quesito com relação aos horários de alimentação, 60\% afirmam realizá-las em tempo regular. Contudo, $76 \%$ efetuam frente à televisão. Com relação ao nível de conhecimento acerca da obesidade e sobrepeso, $68 \%$ atestam não conhecer tais definições. Mas, de forma implícita, teve a preocupação de manter regular o acompanhamento das crianças ao programa de Crescimento e Desenvolvimento, na medida em que, $64 \%$ costumam pesar seus filhos. Ressalta-se a importância quanto à conscientização dos hábitos e as diversas origens que contribuem para a ocorrência da obesidade e sobrepeso.

Descritores: Obesidade pediátrica; Qualidade de Vida; Estilo de vida sedentário

\begin{abstract}
Childhood obesity has presented a considerable increase in the last decades, constituting like a true pandemic. This is a worrying state, since it is a pre-disposition for the development of diseases such as hypertension, diabetes mellitus type 2, cardiovascular diseases, among others. Given this, we sought to highlight the
\end{abstract}


importance of quality of life in the prevention of obesity in minors assisted by a social assistance organization in the city of Itabuna-BA, and specifically to identify the index of children who practice some form of sports, Offered at home and observe the level of knowledge about the subject. Profile tabs were analyzed, in which 25 parents or guardians represented their children. At the end of the research, it was identified that the index of children who practice some form of sport was $84 \%$, with a total of $76 \%$ receiving an incentive to practice sports. Regarding the quality of the food offered by their parents or guardians, in relation to feeding schedules, $60 \%$ affirm that they do it on a regular basis. However, $76 \%$ do so in front of the television. Regarding the level of knowledge about obesity and overweight, $68 \%$ report not knowing such definitions. But implicitly, it was concerned to keep regular monitoring of children to the program of growth and development, as $64 \%$ usually weigh their children. The importance of awareness of habits and the diverse origins that contribute to the occurrence of obesity and overweight is highlighted.

Descriptores: Pediatric obesity; quality of life; Sedentary lifestyle 


\section{INTRODUÇÃO}

As revoluções industrial e tecnológica propiciaram outra grande transformação no cotidiano da maioria das sociedades que é a do consumo alimentar. A busca por opções mais práticas, menos perecíveis, mais baratas vem tornando insalubres os hábitos e a escolha da comida que vai para a mesa. Por se tratar de produtos geralmente mais calóricos ou com adição de quantias elevadas de açúcares, sódio e gorduras saturadas, essa mudança no processo de alimentação vem aumentando a incidência de obesidade e suas doenças correlatas ${ }^{1}$.

Apesar da obesidade não representar nenhuma novidade de caráter científico, o seu atual quadro epidemiológico e de acometimento em todas as idades, a torna um fato que desperta a atenção dos profissionais de saúde, se mostrando como um grave problema de saúde pública, sendo esta uma tendência comum a países desenvolvidos e em desenvolvimento².

O aumento de número de crianças com sobrepeso e obesas está intimamente relacionado com os crescentes gastos públicos em custos diretos com tratamentos hospitalares e doenças crônicas nas unidades básicas de saúde, bem como indiretos, por exemplo, a influência negativa no convívio e vida social. A prática de atividades físicas e a alimentação equilibrada são essenciais para a vida do ser humano em qualquer etapa da vida para a manutenção da saúde, entre as crianças estes hábitos são determinantes para o seu desenvolvimento ${ }^{3}$.

Outra influência direta da revolução tecnológica na obesidade, foi promover uma brusca mudança nas atividades de lazer e trabalho do ser humano, onde o computador, televisão, jogos eletrônicos, celulares com acesso a jogos e internet, entre outras, acabam desestimulando a prática de exercícios físicos, no caso das crianças reduziu em muito as tradicionais brincadeiras que contribuíam para que isto ocorresse naturalmente promovendo o gasto energético necessário ${ }^{4}$.

Em muitos casos o aumento do número de crianças com sobrepeso ou obesas está relacionada com a falta de informação dos pais, responsáveis e, consequentemente, da própria criança. Fatores desencadeadores como alimentação 
inadequada, sedentarismo e ingestão de produtos industrializados ou altamente calóricos devem ser alertados entre a população 5 .

Com isso buscou-se de maneira geral destacar a importância da qualidade de vida na prevenção da obesidade infantil para crianças assistidas por uma entidade de assistência social na cidade de Itabuna-BA, e especificamente Identificar o índice de crianças que praticam alguma modalidade de esporte, conhecer a qualidade da alimentação oferecida em domicílio e observar o nível de conhecimento acerca da obesidade e sobrepeso.

\section{MÉTODOS}

O município de Itabuna fica localizado às margens da BR 101 no estado da Bahia, microrregião Cacaueira, localizada no Sul do estado. Dista de Salvador, capital da Bahia, em $429 \mathrm{~km}$. A área total do município é de 580,49 Km2, e sua área urbana é de 65,93 Km2. É a quinta cidade mais populosa da Bahia, com 218924 habitantes.

A pesquisa utilizou dados secundários obtidos de duas fontes cedidas pela própria instituição que foi objeto de estudo. A priori foi analisada a ficha de acompanhamento nutricional dos alunos também sem as identificações, onde foram selecionadas apenas crianças que se encontravam com IMC elevado, ressalta-se que todos os documentos observados estavam sem as capas para garantia do sigilo. Posteriormente foi feita a análise das fichas socioeconômicas dos pais ou responsáveis de 25 alunos de ambos os sexos, com idade entre 6 e 12 anos, assistidos pela Legião da Boa Vontade (LBV) da cidade de Itabuna-Ba, no período de Maio a Julho de 2015,. também sem as identificações.

Nas fichas foram observadas as seguintes questões socioeconômicas: As crianças praticam esportes ou são incentivadas à prática? As refeições são feitas no horário correto? As refeições são feitas junto à eletro-eletrônicos? Existem obesos na família? Sabe diferenciar obeso de alguém com sobrepeso? Tem costume de pesar seus filhos?

As questões foram escolhidas por estarem diretamente evolvidas no contexto da alimentação e saúde das crianças podendo ter influência direta no estado nutricional das mesmas. 


\section{RESULTADOS}

Com a observação dos 25 questionários socioeconômicos dos pais dos alunos acima do peso matriculados na LBV da cidade de Itabuna, foi possível identificar que $84 \%$ responderam que seus filhos realizavam algum tipo de esporte ao menos duas vezes por semana. Quanto à prática de esportes foi observado que $76 \%$ dos responsáveis pelas crianças indicaram que estimulam a prática de esportes.

Foi possível notar também aos pais quanto à regularidade no horário da alimentação e quinze deles (60\%) informaram que as crianças se alimentavam corretamente nos horários devidos todos os dias, seguidos de nove (36\%) que disseram que não havia estabilidade no horário das refeições, e um dos pais indicou que por passar o dia todo fora de casa não poderia responder corretamente.

Outro hábito que foi observado é o de uso de eletrônicos durante a refeição e $76 \%$ dos pais indicaram que seus filhos fazem as refeições em frente a TVs e computadores com frequência.

Quanto a presença de obesos na família ou dentro de casa a resposta predominante foi que não havia ninguém com essas características. Porém esse dado pode ser contrabalançado com os dados obtidos do conhecimento da diferença de sobrepeso e obesidade, em que $68 \%$ dos pais demonstraram falta de conhecimento do assunto (Tabela 1)

Tabela 1: Presença de obesos na família ou residência e conhecimento a respeito de obesidade e sobrepeso.

\begin{tabular}{lcc}
\hline Presença de obesos & Número de respostas & $\%$ \\
\hline Sim & 8 & 32 \\
Não & 11 & 44 \\
Sem resposta & 6 & 24
\end{tabular}

Conhecimento de 


\section{obesidade}

Sim

Não

Fonte: Do pesquisador.

Nos cuidados referentes ao peso das crianças, $52 \%$ dos responsáveis pelas crianças indicaram que pesam seus filhos com regularidade, outros $12 \%$ responderam que só realizam a pesagem esporadicamente e os demais $36 \%$ afirmaram não ter esse costume.

\section{DISCUSSÃO}

Para a manutenção de uma estrutura corporal saudável, redução de peso, ou até recuperação em processos de doença, o exercício físico, independente do tipo que seja adotado é essencial ${ }^{6}$. Sabendo então que os fatores comportamentais são essenciais no que se refere a pré-disposição à obesidade num infante, é essencial que dentro do seu contexto de vida esteja inserida a atividade física?

A influência dos pais no comportamento infantil é essencial, uma vez que a criança tende a repetir os hábitos observados em seus genitores, então boas práticas alimentares já se fazem como um bom início para a prevenção da obesidade infantil, mas além disso, o estímulo direto às boas práticas como a realização de esportes oriundo dos pais tende a ser bem eficaz inibindo assim 0 comportamento sedentário que é bem comum entre crianças e adolescente atualmente, principalmente nas grandes cidades ${ }^{8}$.

Nessa questão além da influência dos pais, a sociedade civil organizada, o governo, clubes, entre outras entidades também podem atuar para evitar esse quadro preocupante de saúde pública da obesidade infantil, com a promoção de atividades em grupo, segurança em áreas públicas, programas de prática regular de esportes, inclusão em projetos esportivos e divulgação das modalidades disponíveis em suas regiões ${ }^{9}$.

Os desvios de hábitos alimentares são fatores preponderantes no desenvolvimento da obesidade infantil, pois alteram o apetite durante as refeições regulares ou até mesmo inibem a alimentação em horários devidos com uso de 
lanches extremamente calóricos próximos de horário de almoço, ou antes de dormir 6 .

A influência do ambiente é de fundamental importância para que a alimentação do infante seja realizada corretamente, então se os pais usam aparelhos eletrônicos durante as refeições, a criança adquire esse hábito por repetição, e o resultado deste trabalho foi preocupante, pois $76 \%$ dos pais disseram que é uma prática comum às suas crianças. Isto pode alterar horários de sono, desejo por alimentos mais práticos e inapetência pela refeição mais saudável ${ }^{10}$.

Os resultados demonstram que não é necessária apenas a orientação das crianças e sim dos seus educadores em casa que por muitas vezes Ihe falta a informação correta a ser repassada aos filhos e por muitas repetem hábitos incorretos aprendidos em décadas passadas que hoje são de conhecimento geral como inapropriados para o desenvolvimento alimentar de uma criança ${ }^{11}$.

Outro fator em que os pais podem estar influenciando no desenvolvimento da obesidade infantil além da não percepção ou falta de conhecimento a respeito desse quadro é a busca por uma vida prática com alimentação feita principalmente por alimentos industrializados e evitando o que seria julgado como uma perda de tempo, e a baixa inserção de alimentos frescos na dieta dos infantes. Ressalta-se que a publicidade em volta dos ultraprocessados trabalha no intuito de convencer que esses alimentos podem suprir corretamente a dieta para um indivíduo em desenvolvimento ${ }^{12,13}$.

A repercussão familiar do aumento do peso na criança muitas vezes passa desapercebido, por vezes até é estimulada por achar que a criança está comendo pouco e pode ficar desnutrida, isso passa também por tentar mascarar as consequências do quadro de obesidade infantil para criar um ambiente seguro da criança, que por muitas vezes já é motivo de chacota em ambiente escolar ${ }^{14}$. Essa apatia por parte dos genitores perpassa pelo não acompanhamento do estado nutricional dos filhos e deixando assim de tomar cuidados básicos como pesar seus filhos, consequentemente permitindo a evolução de doenças associadas ao aumento do peso ${ }^{15}$.

A falta de cuidado parental em relação à obesidade infantil já é demonstrada em outros estudos que demonstram a falta de pulso na cobrança das autoridades no 
que se trata de espaços para a realização de atividades, o relaxamento quanto a exposição de hipercalóricos aos filhos, os cuidados extremos com a segurança que cerceiam a possibilidade de realização de exercícios em ambientes externos, e o estímulo ao sedentarismo com a apresentação de eletrônicos para diversão em ambiente caseiro ${ }^{16,17}$.

\section{CONCLUSÃO}

O presente estudo demonstra que os pais das crianças matriculadas na escola da Legião da Boa Vontade estão cientes do que é necessário para evitar a obesidade ou sobrepeso em seus filhos, indicando que conhecem os efeitos benéficos de esportes e demais exercícios físicos para seus filhos.

Já no que se trata do outro pilar do bom estado nutricional das crianças que são os hábitos alimentares corretos, foi possível concluir que nessa seara o cuidado já não é tão conhecido e por isso não se faz presente. A ocorrência de comportamentos inadequados tanto na escolha como no modo de alimentar são fatores que propiciam o desenvolvimento da obesidade.

Por fim, conclui-se que a falta de orientação dentro de gerações das famílias está sendo repassada para essas crianças observadas, pois hábitos antigos estão sendo praticados dentro de casa, e isso associado com a superproteção seja ela psicológica para criar um ambiente diferente do social vivido pelo aluno, ou física, no intuito de proteção contra riscos de violência vem contribuindo para 0 desenvolvimento da obesidade infantil.

\section{REFERÊNCIAS}

1- Ferreira S, Tinoco ALA, Panato E, Viana NL. Aspectos etiológicos e o papel do exercício físico na prevenção e controle da obesidade. Revista de educação Física. 2006; 133:15-24.

2- Carvalho EADA, Simão MTJ, Fonseca MC, Andrade RGD, Ferreira MSG, Silva AF, Souza IPRS, Fernandes BS. Obesidade: aspectos epidemiológicos e prevenção. 2013;23(1):74-82. 
3- Cabrera TFC, Correia IFL, Dos Santos, DO, Pacagnelli FL, Prado MTA, Da Silva TD, Monteiro CBM, Fernani DCGL. Análise da prevalência de sobrepeso e obesidade e do nível de atividade física em crianças e adolescentes de uma cidade do sudoeste de São Paulo. Journal of Human Growth and Development. 2014;24(1):67-72.

4- Pedretti A, Pedretti A, Vasconcellos F, Seabra A. O futebol recreativo como uma nova abordagem terapêutica para a obesidade em crianças $\mathrm{e}$ adolescentes: uma revisão. Revista Brasileira de Atividade Física \& Saúde. 2016;21(2):123-132.

5- Santos FDDR, Vitola CB, Arrieira ICO, Silveira-Chagas MC, Gomes GC, Pereira FW. Ações de enfermeiros e professores na prevenção e no combate à obesidade infantil. Northeast Network Nursing Journal. 2014;15(3):123-132.

6- Freitas ASDS, Coelho SC, Ribeiro RL. Obesidade infantil: influência de hábitos alimentares inadequados. Saúde e ambiente. 2009;4(2): 9-14.

7- Oliveira AMAD, Cerqueira EM, Souza JDS, Oliveira ACD. Sobrepeso e obesidade infantil: influência de fatores biológicos e ambientais em Feira de Santana, BA. Arquivo Brasileiro de Endocrinologia Metabólica. 2003;47(2):144-150.

8- Mello EDD, Luft VC, Meyer F. Obesidade infantil: como podemos ser eficazes?. Jornal de pediatria. 2004;80(3):173-182.

9- Silva RCR, Malina RM. Nível de atividade física em adolescentes do Município de Niterói, Rio de Janeiro, Brasil. Caderno de Saúde Pública. 2000;16(4):1091-1097.

10-Triches RM, Giugliani ERJ. Obesidade, práticas alimentares e conhecimentos de nutrição em escolares. Revista de Saúde Pública. 2005;39(4):541-547.

11-Viana V. Psicologia, saúde e nutrição: Contributo para o estudo do comportamento alimentar. Análise Psicológica. 2002;20(4):611-624. 
12-Camargo APPM, Barros-Filho AA, Monteiro AM, Giglio JS. A não percepção da obesidade pode ser um obstáculo no papel das mães de cuidar de seus filhos. Ciência \& Saúde Coletiva. 2013;18(2):323-333.

13-Santos AM, Scherer PT. Mídia e obesidade infantil: uma discussão sobre o peso das propagandas. Revista FAMECOS. 2014;21(1): 208-223.

14-Vive NSDPS. Nem só de pão se vive: a voz das mães na obesidade infantil. Psicologia: ciência e profissão. 2013;33(1): 46-59.

15-Pizzinato A, Dornelles AD, Anton MC. O papel da sociedade e da família na assistência ao sobrepeso e à obesidade infantil: percepção de trabalhadores da saúde em diferentes níveis de atenção. Saúde e Sociedade. 2014;23(4):1275-1287.

16-Mishima-Gomes FKT, Dezan SZ, Barbieri V. Não pode!": a função paterna e a obesidade infantil. Psico. 2014;45(2):176-186.

17-Teixeira VH, Moreira P. Alimentação maternal e status socioeconômico na luta contra a má-nutrição infantil. Jornal de Pediatria. 2016;92(6):546-548. 